\title{
A JOINT UNIVERSALITY THEOREM FOR PERIODIC HURWITZ ZETA-FUNCTIONS
}

\author{
A. JAVTOKAS and A. LAURINČIKAS ${ }^{凶}$
}

(Received 19 July 2007)

Abstract

We prove a joint universality theorem in the Voronin sense for the periodic Hurwitz zeta-functions.

2000 Mathematics subject classification: 11M35, 11M41.

Keywords and phrases: Hurwitz zeta-functions, probability measure, universality, weak convergence.

\section{Introduction}

As usual, denote by $\zeta(s), s=\sigma+i t$, the Riemann zeta-function. In 1975 Voronin discovered in [24] a remarkable universality property for the function $\zeta(s)$. Let $0<r<1 / 4$, and suppose that $f(s)$ is a continuous nonvanishing on the disc $\{s \in \mathbb{C}|| s \mid \leq r\}$ function which is analytic in the interior of this disc. Then he proved that, for every $\varepsilon>0$, there exists a real number $\tau=\tau(\varepsilon)$ such that

$$
\max _{|s| \leq r}|\zeta(s+3 / 4+i \tau)-f(s)|<\varepsilon
$$

Later, the Voronin theorem was improved and generalized for other zeta- and $L$-functions as well as for some classes of Dirichlet series by Bagchi, Bauer, Garbaliauskienè, Garunkštis, Genys, Gonek, Good, Ignatavičiūtė, Kačinskaitè, Matsumoto, Macaitienè, Mishou, Nagoshi, Reich, Schwarz, Steuding, Šleževičienė, the present authors, and others. For a bibliography and results, see the survey papers $[6,13,14,21]$ as well as $[1,10,17,22]$. The latest version of the Voronin theorem is the following. Let, for $T>0$,

$$
v_{T}(\ldots)=\frac{1}{T} \operatorname{meas}\{\tau \in[0, T] \mid \ldots\},
$$

where meas $\{A\}$ denotes the Lebesgue measure of a measurable set $A \subset \mathbb{R}$, and in place of dots a condition satisfied by $\tau$ is to be written. Then we have the following statement, see [10]. Denote, for brevity, $D=\{s \in \mathbb{C} \mid 1 / 2<\sigma<1\}$.

Partially supported by Lithuanian Foundation of Studies and Science.

(C) 2008 Australian Mathematical Society 0004-9727/08 \$A2.00+0.00 
TheOREM A. Suppose that $K$ is a compact subset of the strip D with connected complement, and let $f(s)$ be a continuous function that does not vanish on $K$ and is analytic in the interior of $K$. Then, for every $\varepsilon>0$,

$$
\liminf _{T \rightarrow \infty} \nu_{T}\left(\sup _{s \in K}|\zeta(s+i \tau)-f(s)|<\varepsilon\right)>0 .
$$

So, roughly speaking, by the Voronin theorem, any analytic function is approximated uniformly on compact subsets by shifts of the Riemann zeta-function.

A more complicated problem is the joint approximation of a collection of analytic functions by shifts of zeta- or $L$-functions, Voronin was also the first who observed [25] the joint universality of Dirichlet $L$-functions $L(s, \chi)$. We recall a modern version of a theorem from [25].

TheOREM B. Let $\chi_{1}, \ldots, \chi_{r}$ be pairwise nonequivalent Dirichlet characters, let $K_{1}, \ldots, K_{r}$ be compact subsets of the strip $D$ with connected complements, and let, for each $j=1, \ldots, r, f_{j}(s)$ be a continuous nonvanishing function on $K_{j}$ which is analytic in the interior of $K_{j}$. Then, for every $\varepsilon>0$,

$$
\liminf _{T \rightarrow \infty} \nu_{T}\left(\sup _{1 \leq j \leq r} \sup _{s \in K_{j}}\left|L\left(s+i \tau, \chi_{j}\right)-f_{j}(s)\right|<\varepsilon\right)>0 .
$$

The joint universality of Dirichlet $L$-functions by different methods also was obtained by Gonek [5] and Bagchi [1, 2]. In the proof of Theorem B a certain independence of $L$-functions based on the orthogonality of Dirichlet characters is used essentially. Therefore, this method cannot be applied to obtain the joint universality for other functions.

The joint universality for Lerch zeta-functions with some parameters was obtained in [17]. Some results in the field for Matsumoto zeta-functions are given in [11]. A conditional analogue of Theorem B for zeta-functions of certain cusp forms is proved in [18]. In [4, 12] the joint universality of general Dirichlet series has been investigated. The best results on the generalization of Theorem B are related to twists with Dirichlet characters of some Dirichlet series. This case for Dirichlet series with multiplicative coefficients is considered in [23], while [20] is devoted to automorphic $L$-functions.

The aim of this paper is to obtain a joint universality theorem for periodic Hurwitz zeta-functions. We start with the definition and known results on these functions. Let $\mathfrak{a}=\left\{a_{m} \mid m \in \mathbb{N}_{0}\right\}, \mathbb{N}_{0}=\mathbb{N} \cup\{0\}$, be a periodic sequence of complex numbers with smallest period $k \in \mathbb{N}$, and let $\alpha, 0<\alpha \leq 1$, be a fixed number. The periodic Hurwitz zeta-function $\zeta(s, \alpha ; \mathfrak{a})$ is defined, for $\sigma>1$, by

$$
\zeta(s, \alpha ; \mathfrak{a})=\sum_{m=0}^{\infty} \frac{a_{m}}{(m+\alpha)^{s}} .
$$


If $\mathfrak{a}=\{1\}$, then $\zeta(s, \alpha ; \mathfrak{a})$ becomes the classical Hurwitz zeta-function $\zeta(s, \alpha)$ which has meromorphic continuation to the whole complex plane with a simple pole $s=1$ and residue 1 . It is easily seen that, for $\sigma>1$,

$$
\zeta(s, \alpha ; \mathfrak{a})=\frac{1}{k^{s}} \sum_{l=0}^{k-1} a_{l} \zeta\left(s, \frac{l+\alpha}{k}\right),
$$

and this shows that the function $\zeta(s, \alpha ; \mathfrak{a})$ is analytically continuable to the whole complex plane, except, perhaps, for a simple pole $s=1$. Let

$$
a=\frac{1}{k} \sum_{m=0}^{k-1} a_{m}
$$

If $a=0$, then $\zeta(s, \alpha ; \mathfrak{a})$ is an entire function, and if $a \neq 0$, then $\operatorname{Res} \zeta(s, \alpha ; \mathfrak{a})=a$.

The universality of the function $\zeta(s, \alpha ; \mathfrak{a})$ with transcendental $\alpha$ was studied in [8,9], in [8] the condition $a_{m} \neq 0$, for all $m$, was used, and in [9] this condition was removed.

THEOREM C [9]. Let $\alpha$ be a transcendental number, let $K$ be a compact subset of the strip $D$ with connected complement, and let $f(s)$ be a function that is continuous on $K$ and analytic in the interior of $K$. Then, for every $\varepsilon>0$,

$$
\liminf _{T \rightarrow \infty} \nu_{T}\left(\sup _{s \in K}|\zeta(s+i \tau, \alpha ; \mathfrak{a})-f(s)|<\varepsilon\right)>0 .
$$

We note that in Theorem $\mathrm{C}$, unlike Theorem A, the approximating function $f(s)$ need not be nonvanishing. This is explained by the nonexistence of an Euler product over primes for $\zeta(s, \alpha ; \mathfrak{a})$.

The first results on joint universality for periodic Hurwitz zeta-functions were obtained in [15, 16]. Let $\mathfrak{a}_{j}=\left\{a_{m j} \mid m \in \mathbb{N}_{0}\right\}$ be a periodic sequence of complex numbers with smallest period $k_{j} \in \mathbb{N}, \alpha_{j}, 0<\alpha_{j} \leq 1$, be a fixed number, and, for $\sigma>1$,

$$
\zeta\left(s, \alpha_{j} ; \mathfrak{a}_{j}\right)=\sum_{m=0}^{\infty} \frac{a_{m j}}{\left(m+\alpha_{j}\right)^{s}}, \quad j=1, \ldots, r .
$$

In [15] the case of $k_{1}=\cdots=k_{r}=k$ and $\alpha_{1}=\cdots=\alpha_{r}=\alpha$ has been considered. Let

$$
A=\left(\begin{array}{cccc}
a_{11} & a_{12} & \ldots & a_{1 r} \\
a_{21} & a_{22} & \ldots & a_{2 r} \\
\ldots \ldots & \ldots & \ldots & \ldots \\
a_{k 1} & a_{k 2} & \ldots & a_{k r}
\end{array}\right)
$$

TheOREM D [15]. Suppose that $\alpha$ is transcendental and $\operatorname{rank}(A)=r$. Let, for each $j=1, \ldots, r, K_{j}$ be a compact subset of the strip $D$ with connected complement, and 
let $f_{j}(s)$ be a function that is continuous on $K_{j}$ and analytic in the interior of $K_{j}$. Then, for every $\varepsilon>0$,

$$
\liminf _{T \rightarrow \infty} \nu_{T}\left(\sup _{1 \leq j \leq r} \sup _{s \in K_{j}}\left|\zeta\left(s+i \tau, \alpha ; \mathfrak{a}_{j}\right)-f_{j}(s)\right|<\varepsilon\right)>0 .
$$

In [16] a similar result has been obtained for periodic Hurwitz zeta-functions with different periods $k_{j}$. The aim of this paper is to obtain joint universality for $\zeta\left(s+i \tau, \alpha_{1} ; \mathfrak{a}_{1}\right), \ldots, \zeta\left(s+i \tau, \alpha_{r} ; \mathfrak{a}_{r}\right)$ with $k_{j}$ and $\alpha_{j}$ both different, $j=1, \ldots, r$. We recall that the numbers $a_{1}, \ldots, a_{r}$ are algebraically independent over the field of rational numbers $\mathbb{Q}$ if there is no nonzero rational polynomial $p$ with $p\left(a_{1}, \ldots, a_{r}\right)$ $=0$.

Now, in the definition of the matrix $A$, let $k$ denote the lowest common multiple of $k_{1}, \ldots, k_{r}$.

THEOREM 1. Suppose that the numbers $\alpha_{1}, \ldots, \alpha_{r}$ are algebraically independent over $\mathbb{Q}$, and that $\operatorname{rank}(A)=r$. Let $K_{1}, \ldots, K_{r}$ and $f_{1}(s), \ldots, f_{r}(s)$ be the same as in Theorem $D$. Then, for every $\varepsilon>0$,

$$
\liminf _{T \rightarrow \infty} v_{T}\left(\sup _{1 \leq j \leq r} \sup _{s \in K_{j}}\left|\zeta\left(s+i \tau, \alpha_{j} ; \mathfrak{a}_{j}\right)-f_{j}(s)\right|<\varepsilon\right)>0 .
$$

\section{A limit theorem}

We start the proof of Theorem 1 with a joint limit theorem for the functions $\zeta\left(s, \alpha_{1} ; \mathfrak{a}_{1}\right), \ldots, \zeta\left(s, \alpha_{r} ; \mathfrak{a}_{r}\right)$ in the sense of weak convergence of probability measures in the space of analytic functions. Denote by $H(D)$ the space of functions analytic on $D$ equipped with the topology of uniform convergence on compacta, and let $H^{r}(D)=\underbrace{H(D) \times \cdots \times H(D)}_{r}$. We set $\gamma=\{s \in \mathbb{C}|| s \mid=1\}$, and define

$$
\Omega=\prod_{m=0}^{\infty} \gamma_{m}
$$

where $\gamma_{m}=\gamma$ for all $m \in \mathbb{N}_{0}$. By the Tikhonov theorem, the torus $\Omega$ is a compact topological Abelian group. Hence, the probability Haar measure $m_{H}$ on $(\Omega, \mathcal{B}(\Omega)$ ) $(\mathcal{B}(S)$ denotes the class of Borel sets of the space $S$ ) exists, and we have a probability space $\left(\Omega, \mathcal{B}(\Omega), m_{H}\right)$. Denote by $\omega(m)$ the projection of $\omega \in \Omega$ to the coordinate space $\gamma_{m}$. Moreover, we define $\Omega^{r}=\Omega_{1} \times \cdots \times \Omega_{r}$, where $\Omega_{j}=\Omega$ for $j=1, \ldots, r$. Then $\Omega^{r}$ is also a compact topological Abelian group. Denote by $m_{H r}$ the probability Haar measure on $\left(\Omega^{r}, \mathcal{B}\left(\Omega^{r}\right)\right)$, and on the probability space $\left(\Omega^{r}, \mathcal{B}\left(\Omega^{r}\right), m_{H r}\right)$ define an $H^{r}(D)$-valued random element $\zeta(s, \omega)$ by

$$
\zeta(s, \boldsymbol{\alpha}, \boldsymbol{\omega} ; \mathfrak{a})=\left(\zeta\left(s, \alpha_{1}, \omega_{1} ; \mathfrak{a}_{1}\right), \ldots, \zeta\left(s, \alpha_{r}, \omega_{r} ; \mathfrak{a}_{r}\right)\right),
$$


where

$$
\zeta\left(s, \alpha_{j}, \omega_{j} ; \mathfrak{a}_{j}\right)=\sum_{m=0}^{\infty} \frac{a_{m j} \omega_{j}(m)}{\left(m+\alpha_{j}\right)^{s}},
$$

$\omega_{j} \in \Omega, j=1, \ldots, r, \boldsymbol{\alpha}=\left(\alpha_{1}, \ldots, \alpha_{r}\right), \mathfrak{a}=\left(\mathfrak{a}_{1}, \ldots, \mathfrak{a}_{r}\right)$ and $\boldsymbol{\omega}=\left(\omega_{1}, \ldots, \omega_{r}\right)$. Let $P_{\zeta}$ denote the distribution of the random element $\zeta(s, \omega)$, that is,

$$
P_{\zeta}(A)=m_{H r}\left(\boldsymbol{\omega} \in \Omega^{r}: \zeta(s, \boldsymbol{\alpha}, \boldsymbol{\omega} ; \mathfrak{a}) \in A\right), \quad A \in \mathcal{B}\left(H^{r}(D)\right),
$$

and define the probability measure

$$
P_{T}(A)=v_{T}\left(\left(\zeta\left(s+i \tau, \alpha_{1} ; \mathfrak{a}_{1}\right), \ldots, \zeta\left(s+i \tau, \alpha_{r} ; \mathfrak{a}_{r}\right)\right) \in A\right), \quad A \in \mathcal{B}\left(H^{r}(D)\right) .
$$

THEOREM 2. Suppose that $\alpha_{1}, \ldots, \alpha_{r}$ are algebraically independent over $\mathbb{Q}$. Then the probability measure $P_{T}$ converges weakly to $P_{\zeta}$ as $T \rightarrow \infty$.

We begin with a limit theorem for probability measures on $\left(\Omega^{r}, \mathcal{B}\left(\Omega^{r}\right)\right)$. Define

$$
\begin{array}{r}
Q_{T, r}(A)=v_{T}\left(\left(\left(\left(m+\alpha_{1}\right)^{-i \tau}: m \in \mathbb{N}_{0}\right), \ldots,\left(\left(m+\alpha_{r}\right)^{-i \tau}: m \in \mathbb{N}_{0}\right)\right) \in A\right), \\
A \in \mathcal{B}\left(\Omega^{r}\right) .
\end{array}
$$

Lemma 3. Suppose that $\alpha_{1}, \ldots, \alpha_{r}$ are algebraically independent over $\mathbb{Q}$. Then the probability measure $Q_{T, r}$ converges weakly to the Haar measure $m_{H r}$ on $\left(\Omega^{r}, \mathcal{B}\left(\Omega^{r}\right)\right)$ as $T \rightarrow \infty$.

PROOF. We use a method of Fourier transforms. The dual group of $\Omega^{r}$ is

$$
\bigoplus_{j=1}^{r} \bigoplus_{m=0}^{\infty} \mathbb{Z}_{m j}
$$

where $\mathbb{Z}_{m j}=\mathbb{Z}=\{\ldots,-1,0,1, \ldots\}$ for all $m \in \mathbb{N}_{0}$ and $j=1, \ldots, r .\left(\mathbf{k}_{1}, \ldots, \mathbf{k}_{r}\right)$ $=\left(k_{01}, k_{11}, \ldots, k_{0 r}, k_{1 r}, \ldots\right) \in \bigoplus_{j=1}^{r} \bigoplus_{m=0}^{\infty} \mathbb{Z}_{m j}$, where only a finite number of integers $k_{m j}, m \in \mathbb{N}_{0}, j=1, \ldots, r$, are distinct from zero, acts on $\Omega^{r}$ by

$$
\left(\mathbf{x}_{1}, \ldots, \mathbf{x}_{r}\right) \rightarrow\left(\mathbf{x}_{1}^{\mathbf{k}_{1}}, \ldots, \mathbf{x}_{r}^{\mathbf{k}_{r}}\right)=\prod_{j=1}^{r} \prod_{m=0}^{\infty} x_{m j}^{k_{m j}},
$$

where $\mathbf{x}_{j}=\left(x_{0 j}, x_{1 j}, \ldots\right), x_{m j} \in \gamma, m \in \mathbb{N}_{0}, j=1, \ldots, r$. Hence we obtain that the Fourier transform $g_{T, r}\left(\mathbf{k}_{1}, \ldots, \mathbf{k}_{r}\right)$ of the probability measure $Q_{T, r}$ is of the form

$$
\begin{aligned}
g_{T, r}\left(\mathbf{k}_{1}, \ldots, \mathbf{k}_{r}\right) & =\int_{\Omega^{r}} \prod_{j=1}^{r} \prod_{m=0}^{\infty} x_{m j}^{k_{m j}} d Q_{T, r} \\
& =\frac{1}{T} \int_{0}^{T} \prod_{j=1}^{r} \prod_{m=0}^{\infty} e^{-i \tau k_{m j} \log \left(m+\alpha_{j}\right)} d \tau \\
& =\frac{1}{T} \int_{0}^{T} \exp \left\{-i \tau \sum_{j=1}^{r} \sum_{m=0}^{\infty} k_{m j} \log \left(m+\alpha_{j}\right)\right\} d \tau,
\end{aligned}
$$


where only a finite number of integers $k_{m j}, m \in \mathbb{N}_{0}, j=1, \ldots, r$, are distinct from zero. In virtue of the algebraic independence of $\alpha_{1}, \ldots, \alpha_{r}$, for $\left(\mathbf{k}_{1}, \ldots, \mathbf{k}_{r}\right) \neq$ $(\mathbf{0}, \ldots, \mathbf{0})$,

$$
\prod_{j=1}^{r} \prod_{m=0}^{\infty}\left(m+\alpha_{j}\right)^{k_{m j}} \neq 1
$$

therefore,

$$
\sum_{j=1}^{r} \sum_{m=0}^{\infty} k_{m j} \log \left(m+\alpha_{j}\right) \neq 0 .
$$

Thus, by (1)

$$
\begin{aligned}
& g_{T, r}\left(\mathbf{k}_{1}, \ldots, \mathbf{k}_{r}\right) \\
& \quad= \begin{cases}1 & \text { if }\left(\mathbf{k}_{1}, \ldots, \mathbf{k}_{r}\right)=(\mathbf{0}, \ldots, \mathbf{0}), \\
\frac{\exp \left\{-i T \sum_{j=1}^{r} \sum_{m=0}^{\infty} k_{m j} \log \left(m+\alpha_{j}\right)\right\}-1}{-i T \sum_{j=1}^{r} \sum_{m=0}^{\infty} k_{m j} \log \left(m+\alpha_{j}\right)} & \text { if }\left(\mathbf{k}_{1}, \ldots, \mathbf{k}_{r}\right) \neq(\mathbf{0}, \ldots, \mathbf{0}),\end{cases}
\end{aligned}
$$

and

$$
\lim _{T \rightarrow \infty} g_{T, r}\left(\mathbf{k}_{1}, \ldots, \mathbf{k}_{r}\right)= \begin{cases}1 & \text { if }\left(\mathbf{k}_{1}, \ldots, \mathbf{k}_{r}\right)=(\mathbf{0}, \ldots, \mathbf{0}), \\ 0 & \text { if }\left(\mathbf{k}_{1}, \ldots, \mathbf{k}_{r}\right) \neq(\mathbf{0}, \ldots, \mathbf{0}) .\end{cases}
$$

This and [7, Theorem 1.4.2] show that the measure $Q_{T, r}$ converges weakly to $m_{H r}$ as $T \rightarrow \infty$.

For $\sigma_{1}>1 / 2$ and $m, n \in \mathbb{N}_{0}$, define

$$
v_{j}(m, n)=\exp \left\{-\left(\frac{m+\alpha_{j}}{n+\alpha_{j}}\right)^{\sigma_{1}}\right\}, \quad j=1, \ldots, r .
$$

Moreover, for $N \in \mathbb{N}_{0}, s \in D$ and fixed $\widehat{\omega}_{j} \in \Omega$, we set

$$
\zeta_{N, n, j}\left(s, \alpha_{j} ; \mathfrak{a}_{j}\right)=\sum_{m=0}^{N} \frac{a_{m j} v_{j}(m, n)}{\left(m+\alpha_{j}\right)^{s}},
$$

and

$$
\zeta_{N, n, j}\left(s, \alpha_{j}, \widehat{\omega}_{j} ; \mathfrak{a}_{j}\right)=\sum_{m=0}^{N} \frac{a_{m j} \widehat{\omega}_{j}(m) v_{j}(m, n)}{\left(m+\alpha_{j}\right)^{s}}, \quad j=1, \ldots, r,
$$

and define the probability measures

$$
\begin{array}{r}
P_{T, N, n}(A)=v_{T}\left(\left(\zeta_{N, n, 1}\left(s+i \tau, \alpha_{1} ; \mathfrak{a}_{1}\right), \ldots, \zeta_{N, n, r}\left(s+i \tau, \alpha_{r} ; \mathfrak{a}_{r}\right)\right) \in A\right), \\
A \in \mathcal{B}\left(H^{r}(D)\right),
\end{array}
$$

and

$$
\begin{array}{r}
\widehat{P}_{T, N, n}(A)=v_{T}\left(\left(\zeta_{N, n, 1}\left(s+i \tau, \alpha_{1}, \widehat{\omega}_{1} ; \mathfrak{a}_{1}\right), \ldots, \zeta_{N, n, r}\left(s+i \tau, \alpha_{r}, \widehat{\omega}_{r} ; \mathfrak{a}_{r}\right)\right) \in A\right), \\
A \in \mathcal{B}\left(H^{r}(D)\right) .
\end{array}
$$


THEOREM 4. The probability measures $P_{T, N, n}$ and $\widehat{P}_{T, N, n}$ both converge weakly to the same probability measure on $\left(H^{r}(D), \mathcal{B}\left(H^{r}(D)\right)\right)$ as $T \rightarrow \infty$.

PROOF. Define a function $u: \Omega^{r} \rightarrow H^{r}(D)$ by the formula

$$
u\left(\omega_{1}, \ldots, \omega_{r}\right)=\left(\zeta_{N, n, 1}\left(s, \alpha_{1}, \omega_{1} ; \mathfrak{a}_{1}\right), \ldots, \zeta_{N, n, r}\left(s, \alpha_{r}, \omega_{r} ; \mathfrak{a}_{r}\right)\right)
$$

$\left(\omega_{1}, \ldots, \omega_{r}\right) \in \Omega^{r}$. Obviously, the function $u$ is continuous, moreover,

$$
\begin{aligned}
& u\left(\left(\left(m+\alpha_{1}\right)^{-i \tau}: m \in \mathbb{N}_{0}\right), \ldots,\left(\left(m+\alpha_{r}\right)^{-i \tau}: m \in \mathbb{N}_{0}\right)\right) \\
& \quad=\left(\zeta_{N, n, 1}\left(s+i \tau, \alpha_{1} ; \mathfrak{a}_{1}\right), \ldots, \zeta_{N, n, r}\left(s+i \tau, \alpha_{r} ; \mathfrak{a}_{r}\right)\right) .
\end{aligned}
$$

Hence, $P_{T, N, n}=Q_{T, r} u^{-1}$, and Lemma 3 together with [3, Theorem 5.1] imply the weak convergence of $P_{T, N, n}$ to $m_{H r} u^{-1}$ as $T \rightarrow \infty$.

For the proof of the weak convergence of $\widehat{P}_{T, N, n}$, define $u_{1}: \Omega^{r} \rightarrow \Omega^{r}$ by

$$
u_{1}\left(\omega_{1}, \ldots, \omega_{r}\right)=\left(\omega_{1} \widehat{\omega}_{1}, \ldots, \omega_{r} \widehat{\omega}_{r}\right) .
$$

Then we have that

$$
\begin{aligned}
& \left(\zeta_{N, n, 1}\left(s+i \tau, \alpha_{1}, \widehat{\omega}_{1} ; \mathfrak{a}_{1}\right), \ldots, \zeta_{N, n, r}\left(s+i \tau, \alpha_{r}, \widehat{\omega}_{r} ; \mathfrak{a}_{r}\right)\right) \\
& \quad=u\left(u_{1}\left(\left(\left(m+\alpha_{1}\right)^{-i \tau}: m \in \mathbb{N}_{0}\right), \ldots,\left(\left(m+\alpha_{r}\right)^{-i \tau}: m \in \mathbb{N}_{0}\right)\right)\right) .
\end{aligned}
$$

This, in a similar manner to the above, shows that the measure $\widehat{P}_{T, N, n}$ converges weakly to $m_{H r}\left(u u_{1}\right)^{-1}$ as $T \rightarrow \infty$. However, the invariance of the Haar measure $m_{H r}$ gives the equality

$$
m_{H r}\left(u u_{1}\right)^{-1}=\left(m_{H r} u_{1}^{-1}\right) u^{-1}=m_{H r} u^{-1} .
$$

The theorem is proved.

Now define

$$
\zeta_{n, j}\left(s, \alpha_{j} ; \mathfrak{a}_{j}\right)=\sum_{m=0}^{\infty} \frac{a_{m j} v_{j}(m, n)}{\left(m+\alpha_{j}\right)^{s}}, \quad j=1, \ldots, r .
$$

It is not difficult to see that the series converges absolutely for $\sigma>1 / 2$. Further, for $\omega_{j} \in \Omega$, let

$$
\zeta_{n, j}\left(s, \alpha_{j}, \omega_{j} ; \mathfrak{a}_{j}\right)=\sum_{m=0}^{\infty} \frac{a_{m j} \omega_{j}(m) v_{j}(m, n)}{\left(m+\alpha_{j}\right)^{s}}, \quad j=1, \ldots, r,
$$

since $\left|\omega_{j}(m)\right|=1$, the series also being absolutely convergent for $\sigma>1 / 2$. Our next aim is the weak convergence of the following probability measures

$$
\begin{array}{r}
P_{T, n}(A)=v_{T}\left(\left(\zeta_{n, 1}\left(s+i \tau, \alpha_{1} ; \mathfrak{a}_{1}\right), \ldots, \zeta_{n, r}\left(s+i \tau, \alpha_{r} ; \mathfrak{a}_{r}\right)\right) \in A\right), \\
A \in \mathcal{B}\left(H^{r}(D)\right),
\end{array}
$$


and

$$
\begin{array}{r}
\widehat{P}_{T, n}(A)=\nu_{T}\left(\zeta_{n, 1}\left(s+i \tau, \alpha_{1}, \omega_{1} ; \mathfrak{a}_{1}\right), \ldots, \zeta_{n, r}\left(s+i \tau, \alpha_{r}, \omega_{r} ; \mathfrak{a}_{r}\right)\right), \\
A \in \mathcal{B}\left(H^{r}(D)\right)
\end{array}
$$

THEOREM 5. The probability measures $P_{T, n}$ and $\widehat{P}_{T, n}$ both converge weakly to the same probability measure on $\left(H^{r}(D), \mathcal{B}\left(H^{r}(D)\right)\right)$ as $T \rightarrow \infty$.

PROOF. We begin with the definition of a metric on $H^{r}(D)$. Let $\left\{K_{n}\right\}$ be a sequence of compact subsets of the strip $D$ such that $K_{n} \subset K_{n+1}, D=\bigcup_{n=1}^{\infty} K_{n}$, and if $K$ is a compact subset of $D$, then $K \subseteq K_{n}$ for some $n$. Then define, for $f, g \in H(D)$,

$$
\varrho(f, g)=\sum_{n=1}^{\infty} 2^{-n} \frac{\varrho_{n}(f, g)}{1+\varrho_{n}(f, g)},
$$

where $\varrho_{n}(f, g)=\sup _{s \in K_{n}}|f(s)-g(s)|$. Then $\varrho(f, g)$ is a metric on $H(D)$ which induces its topology of uniform convergence on compacta. Now, taking, for $\mathbf{f}=\left(f_{1}, \ldots, f_{r}\right), \mathbf{g}=\left(g_{1}, \ldots, g_{r}\right) \in H^{r}(D)$,

$$
\varrho^{(r)}(\mathbf{f}, \mathbf{g})=\max _{1 \leq j \leq r} \varrho\left(f_{j}, g_{j}\right)
$$

we have a metric on $H^{r}(D)$ inducing its topology.

By Theorem 4, the probability measures $P_{T, N, n}$ and $\widehat{P}_{T, N, n}$ both converge weakly to the same probability measure, say, $P_{N, n}$ as $T \rightarrow \infty$. Our first aim is to prove that, for fixed $n$, the family $\left\{P_{N, n} \mid N \in \mathbb{N}_{0}\right\}$ is tight. Let $\theta$ be a random variable defined on the probability space $([0,1], \mathcal{B}([0,1]), \mathbb{P})$ with the Lebesgue measure $\mathbb{P}$, and uniformly distributed on $[0,1]$. Let, for $j=1, \ldots, r$,

$$
X_{T, N, n, j}(s)=\zeta_{N, n, j}\left(s+i T \theta, \alpha_{j} ; \mathfrak{a}_{j}\right),
$$

and

$$
\mathbf{X}_{T, N, n}(s)=\left(X_{T, N, n, 1}(s), \ldots, X_{T, N, n, r}(s)\right)
$$

Since $P_{T, N, n}$ is the distribution of $\mathbf{X}_{T, N, n}(s)$, we have that

$$
\mathbf{X}_{T, N, n}(s) \underset{T \rightarrow \infty}{\stackrel{\mathcal{D}}{\longrightarrow}} \mathbf{X}_{N, n}(s)
$$

where $\mathbf{X}_{N, n}(s)=\left(X_{N, n, 1}(s), \ldots, X_{N, n, r}(s)\right)$ is an $H^{r}(D)$-valued random element having the distribution $P_{N, n}$, and $\stackrel{\mathcal{D}}{\rightarrow}$, as usual, denotes the convergence in distribution. 
The series for $\zeta_{n, j}\left(s, \alpha_{j} ; \mathfrak{a}_{j}\right), j=1, \ldots, r$, converges absolutely for $\sigma>1 / 2$, and uniformly on compact subsets of $D$. Hence, for $M_{l j}>0, j=1, \ldots, r, l \in \mathbb{N}$,

$$
\begin{aligned}
& \limsup _{T \rightarrow \infty} \mathbb{P}\left(\sup _{s \in K_{l}}\left|X_{T, N, n, j}(s)\right|>M_{l j} \text { for some } j\right) \\
& \quad \leq \sum_{j=1}^{r} \limsup _{T \rightarrow \infty} \mathbb{P}\left(\sup _{s \in K_{l}}\left|X_{T, N, n, j}(s)\right|>M_{l j}\right) \\
& \quad \leq \sum_{j=1}^{r} \frac{1}{M_{l j}} \sup _{N \geq 1} \limsup _{T \rightarrow \infty} \frac{1}{T} \int_{0}^{T} \sup _{s \in K_{l}}\left|\zeta_{N, n, j}\left(s+i \tau, \alpha_{j} ; \mathfrak{a}_{j}\right)\right| d \tau \\
& \quad \leq \sum_{j=1}^{r} \frac{R_{l j}}{M_{l j}}
\end{aligned}
$$

where $R_{l j}<\infty$ for all $j=1, \ldots, r, l \in \mathbb{N}$. If $M_{l j}=R_{l j} 2^{l} r / \varepsilon$, then this yields

$$
\limsup _{T \rightarrow \infty} \mathbb{P}\left(\sup _{s \in K_{l}}\left|X_{T, N, n, j}(s)\right|>M_{l j} \text { for some } j\right) \leq \frac{\varepsilon}{2^{l}} \text {. }
$$

Hence, the continuity of probability and (2) show that, for all $l \in \mathbb{N}$,

$$
\mathbb{P}\left(\sup _{s \in K_{l}}\left|X_{T, N, n, j}(s)\right|>M_{l j} \text { for some } j\right) \leq \frac{\varepsilon}{2^{l}} .
$$

Now we set

$$
H_{\varepsilon}^{r}=\left\{\left(f_{1}, \ldots, f_{r}\right) \in H^{r}(D)\left|\sup _{s \in K_{l}}\right| f_{j}(s) \mid \leq M_{l j}, j=1, \ldots, r, l \in \mathbb{N}\right\} .
$$

Since the set $H_{\varepsilon}^{r}$ is uniformly bounded on compact subsets of $D$, it is compact in $H^{r}(D)$. Moreover, in view of (3), for all $N \in \mathbb{N}_{0}$,

$$
\mathbb{P}\left(\mathbf{X}_{N, n}(s) \in H_{\varepsilon}^{r}\right) \geq 1-\varepsilon .
$$

Hence, the definition of the random element $\mathbf{X}_{N, n}(s)$ implies

$$
P_{N, n}\left(H_{\varepsilon}^{r}\right) \geq 1-\varepsilon, \quad N \in \mathbb{N}_{0} .
$$

This means that the family of probability measures $\left\{P_{N, n} \mid N \in \mathbb{N}_{0}\right\}$ is tight.

For $j=1, \ldots, r$, we have the following result which holds uniformly on compact subsets of the strip $D$

$$
\lim _{N \rightarrow \infty} \zeta_{N, n, j}\left(s, \alpha_{j} ; \mathfrak{a}_{j}\right)=\zeta_{n, j}\left(s, \alpha_{j} ; \mathfrak{a}_{j}\right)
$$


Hence, using the Chebyshev inequality, we find that, for every $\varepsilon>0$,

$$
\begin{aligned}
& \lim _{N \rightarrow \infty} \limsup _{T \rightarrow \infty} v_{T}\left(\varrho^{(r)}\left(\zeta_{N, n}(s, \boldsymbol{\alpha} ; \mathfrak{a}), \zeta_{n}(s, \boldsymbol{\alpha} ; \mathfrak{a})\right) \geq \varepsilon\right) \\
& \quad \leq \lim _{N \rightarrow \infty} \limsup _{T \rightarrow \infty} \frac{1}{T \varepsilon} \sum_{j=1}^{r} \int_{0}^{T} \varrho\left(\zeta_{N, n}(s+i \tau, \boldsymbol{\alpha} ; \mathfrak{a}), \zeta_{n}(s+i \tau, \boldsymbol{\alpha} ; \mathfrak{a})\right) d \tau=0,
\end{aligned}
$$

Here $\zeta_{N, n}(s, \boldsymbol{\alpha} ; \mathfrak{a})$ and $\zeta_{n}(s, \boldsymbol{\alpha} ; \mathfrak{a})$ denote the vectors $\left(\zeta_{N, n}\left(s, \alpha_{1} ; \mathfrak{a}_{1}\right), \ldots\right.$, $\left.\zeta_{N, n}\left(s, \alpha_{r} ; \mathfrak{a}_{r}\right)\right)$ and $\left(\zeta_{n}\left(s, \alpha_{1} ; \mathfrak{a}_{1}\right), \ldots, \zeta_{n}\left(s, \alpha_{r} ; \mathfrak{a}_{r}\right)\right)$, respectively.

Now, let

$$
\mathbf{X}_{T, n}(s)=\left(X_{T, n, 1}(s), \ldots, X_{T, n, r}(s)\right),
$$

where

$$
X_{T, n, j}(s)=\zeta_{n, j}\left(s+i T \theta, \alpha_{j} ; \mathfrak{a}_{j}\right), \quad j=1, \ldots, r .
$$

Then, taking into account (4), we obtain that

$$
\lim _{N \rightarrow \infty} \limsup _{T \rightarrow \infty} \mathbb{P}\left(\varrho^{(r)}\left(\mathbf{X}_{T, n}(s), \mathbf{X}_{T, N, n}(s)\right) \geq \varepsilon\right)=0 .
$$

As we have seen above, the family of probability measures $\left\{P_{N, n} \mid N \in \mathbb{N}_{0}\right\}$ is tight, therefore by the Prokhorov theorem (see, for example, [3]) it is relatively compact. Hence, there exists a subsequence $\left\{P_{N_{1}, n}\right\} \subset\left\{P_{N, n}\right\}$ such that the measure $P_{N_{1}, n}$ converges weakly to the measure $P_{n}$, say, as $N_{1} \rightarrow \infty$. Then, by the definition of the vector $\mathbf{X}_{N, n}(s)$

$$
\mathbf{X}_{N_{1}, n}(s) \underset{N_{1} \rightarrow \infty}{\stackrel{\mathcal{D}}{\longrightarrow}} P_{n}
$$

Since the space $H^{r}(D)$ is separable, (2), (5), (6) and [3, Theorem 4.2] show that

$$
\mathbf{X}_{T, n}(s) \underset{T \rightarrow \infty}{\stackrel{\mathcal{D}}{\longrightarrow}} P_{n},
$$

in other words, the measure $P_{T, n}$ converges weakly to $P_{n}$ as $T \rightarrow \infty$.

In view of (7) we have that the measure $P_{n}$ is independent of the subsequence $\left\{P_{N_{1}, n}\right\}$. Thus, in view of the relative compactness of $\left\{P_{N, n} \mid N \in \mathbb{N}_{0}\right\}$ and [3, Theorem 2.3],

$$
\mathbf{X}_{N, n}(s) \underset{N \rightarrow \infty}{\stackrel{\mathcal{D}}{\longrightarrow}} P_{n}
$$

Define

$$
\widehat{\mathbf{X}}_{T, N, n}(s)=\left(\zeta_{N, n, 1}\left(s+i T \theta, \alpha_{1}, \omega_{1} ; \mathfrak{a}_{1}\right), \ldots, \zeta_{N, n, r}\left(s+i T \theta, \alpha_{r}, \omega_{r} ; \mathfrak{a}_{r}\right)\right),
$$

and

$$
\widehat{\mathbf{X}}_{T, n}(s)=\left(\zeta_{n, 1}\left(s+i T \theta, \alpha_{1}, \omega_{1} ; \mathfrak{a}_{1}\right), \ldots, \zeta_{n, r}\left(s+i T \theta, \alpha_{r}, \omega_{r} ; \mathfrak{a}_{r}\right)\right)
$$


Then, repeating the above arguments for the vectors $\widehat{\mathbf{X}}_{T, N, n}(s)$ and $\widehat{\mathbf{X}}_{T, n}(s)$, and using relation (8), we obtain that the measure $\widehat{P}_{T, n}$ also converges weakly to $P_{n}$ as $T \rightarrow \infty$.

Proof of Theorem 2. On $\left(H^{r}(D), \mathcal{B}\left(H^{r}(D)\right)\right)$, define one more probability measure

$$
\widehat{P}_{T}(A)=v_{T}\left(\left(\zeta\left(s+i \tau, \alpha_{1}, \omega_{1} ; \mathfrak{a}_{1}\right), \ldots, \zeta\left(s+i \tau, \alpha_{r}, \omega_{r} ; \mathfrak{a}_{r}\right)\right) \in A\right) .
$$

We divide the proof of Theorem 2 into two parts. In the first part we prove that the probability measures $P_{T}$ and $\widehat{P}_{T}$ both converge weakly to the same measure $P$, say, and in the second part we identify the measure $P$. In the first part we use the same method as in the proof of Theorem 5 .

By Theorem 5, the probability measures $P_{T, n}$ and $\widehat{P}_{T, n}$ both converge weakly to the measure $P_{n}$ as $T \rightarrow \infty$. Since $P_{T, n}$ is the distribution of $\mathbf{X}_{T, N, n}(s)$, we have from this that

$$
\mathbf{X}_{T, n}(s) \underset{T \rightarrow \infty}{\stackrel{\mathcal{D}}{\longrightarrow}} \mathbf{X}_{n}(s)
$$

where $\mathbf{X}_{T, n}(s)=\left(X_{n, 1}(s), \ldots, X_{n, r}(s)\right)$ is an $H^{r}(D)$-valued random element having the distribution $P_{n}$.

Since the series for $\zeta_{n, j}\left(s, \alpha_{j} ; \mathfrak{a}_{j}\right)$ converges absolutely for $\sigma>1 / 2$, we find that, for $\sigma>1 / 2$,

$$
\begin{array}{r}
\int_{0}^{T}\left|\zeta_{n, j}\left(\sigma+i t, \alpha_{j} ; \mathfrak{a}_{j}\right)\right|^{2} d t=O\left(T \sum_{m=0}^{\infty} \frac{\left|a_{m j}\right|^{2} v^{2}(m, n)}{(m+\alpha)^{2 \sigma}}\right)=O(T) \\
j=1, \ldots, r .
\end{array}
$$

Hence, using the Cauchy integral formula, we obtain that there exists $R_{l j}<\infty$ such that

$$
\begin{array}{r}
\limsup _{T \rightarrow \infty} \frac{1}{T} \int_{0}^{T} \sup _{s \in K_{l}}\left|\zeta_{n, j}\left(\sigma+i \tau, \alpha_{j} ; \mathfrak{a}_{j}\right)\right|^{2} d \tau \leq R_{l j}, \\
j=1, \ldots, r, l \in \mathbb{N} .
\end{array}
$$

We take $M_{l j}=R_{l j} 2^{l} r / \varepsilon$. Then (10) together with Chebyshev's inequality implies

$$
\limsup _{T \rightarrow \infty} \mathbb{P}\left(\sup _{s \in K_{l}}\left|X_{T, n, j}(s)\right|>M_{l j} \text { for some } j\right) \leq \frac{\varepsilon}{2^{l}}, \quad l \in \mathbb{N} .
$$

This and (9) yield

$$
\mathbb{P}\left(\sup _{s \in K_{l}}\left|X_{n, j}(s)\right|>M_{l j} \text { for some } j\right) \leq \frac{\varepsilon}{2^{l}}, \quad l \in \mathbb{N} .
$$


Hence, it follows that

$$
P_{n}\left(H_{\varepsilon}^{r}\right) \geq 1-\varepsilon
$$

for all $n \in \mathbb{N}_{0}$. Thus, we have proved that the family of probability measures $\left\{P_{n} \mid n \in\right.$ $\mathbb{N}_{0}$ \} is tight, and by the Prokhorov theorem it is relatively compact.

Since the numbers $\alpha_{1}, \ldots, \alpha_{r}$ are algebraically independent over $\mathbb{Q}$, clearly, they are transcendental. Then in [9] it was obtained that

$$
\lim _{n \rightarrow \infty} \limsup _{T \rightarrow \infty} \frac{1}{T} \int_{0}^{T} \varrho\left(\zeta\left(s+i \tau, \alpha_{j} ; \mathfrak{a}_{j}\right), \zeta_{n, j}\left(s+i \tau, \alpha_{j} ; \mathfrak{a}_{j}\right)\right) d \tau=0,
$$

and, for almost all $\omega_{j} \in \Omega$,

$$
\lim _{n \rightarrow \infty} \limsup _{T \rightarrow \infty} \frac{1}{T} \int_{0}^{T} \varrho\left(\zeta\left(s+i \tau, \alpha_{j}, \omega_{j} ; \mathfrak{a}_{j}\right), \zeta_{n, j}\left(s+i \tau, \alpha_{j}, \omega_{j} ; \mathfrak{a}_{j}\right)\right) d \tau=0,
$$

$j=1, \ldots, r$. Hence, denoting by $\zeta(s, \boldsymbol{\alpha} ; \mathfrak{a})$ the vector $\left(\zeta\left(s, \alpha_{1} ; \mathfrak{a}_{1}\right), \ldots\right.$, $\left.\zeta\left(s, \alpha_{r} ; \mathfrak{a}_{r}\right)\right)$, and similarly defining the vectors $\zeta(s, \boldsymbol{\alpha}, \boldsymbol{\omega} ; \mathfrak{a})$ and $\zeta_{n}(s, \boldsymbol{\alpha}, \boldsymbol{\omega} ; \mathfrak{a})$, we deduce from the last two relations that

$$
\lim _{n \rightarrow \infty} \limsup _{T \rightarrow \infty} \frac{1}{T} \int_{0}^{T} \varrho^{(r)}\left(\zeta(s+i \tau, \boldsymbol{\alpha} ; \mathfrak{a}), \zeta_{n}(s+i \tau, \boldsymbol{\alpha} ; \mathfrak{a}) d \tau=0,\right.
$$

and, for almost all $\left(\omega_{1}, \ldots, \omega_{r}\right) \in \Omega^{r}$,

$$
\lim _{n \rightarrow \infty} \limsup _{T \rightarrow \infty} \frac{1}{T} \int_{0}^{T} \varrho^{(r)}\left(\zeta(s+i \tau, \boldsymbol{\alpha}, \boldsymbol{\omega} ; \mathfrak{a}), \zeta_{n}(s+i \tau, \boldsymbol{\alpha}, \boldsymbol{\omega} ; \mathfrak{a}) d \tau=0 .\right.
$$

Now we define

$$
\mathbf{X}_{T}(s)=\left(X_{T, 1}(s), \ldots, X_{T, r}(s)\right)
$$

where

$$
X_{T, j}(s)=\zeta\left(s+i T \theta, \alpha_{j} ; \mathfrak{a}_{j}\right), \quad j=1, \ldots, r .
$$

Then by (11), for every $\varepsilon>0$,

$$
\begin{aligned}
& \lim _{n \rightarrow \infty} \limsup _{T \rightarrow \infty} v_{T}\left(\varrho^{(r)}\left(\zeta(s+i \tau, \boldsymbol{\alpha} ; \mathfrak{a}), \zeta_{n}(s+i \tau, \boldsymbol{\alpha} ; \mathfrak{a})\right)\right. \\
& \quad \leq \frac{1}{\varepsilon T} \int_{0}^{T} \varrho^{(r)}\left(\zeta(s+i \tau, \boldsymbol{\alpha} ; \mathfrak{a}), \zeta_{n}(s+i \tau, \boldsymbol{\alpha} ; \mathfrak{a})\right) d \tau=0,
\end{aligned}
$$

and, therefore,

$$
\lim _{n \rightarrow \infty} \limsup _{T \rightarrow \infty} \mathbb{P}\left(\varrho^{(r)}\left(\mathbf{X}_{T}(s), \mathbf{X}_{T, n}(s)\right) \geq \varepsilon\right)=0 .
$$

In virtue of the relative compactness of $\left\{P_{n} \mid n \in \mathbb{N}_{0}\right\}$, we can find $\left\{P_{n_{1}}\right\} \subset\left\{P_{n}\right\}$ such that $P_{n_{1}}$ converges weakly, say, to $P$ as $n_{1} \rightarrow \infty$. Hence,

$$
\mathbf{X}_{n_{1}}(s) \underset{n_{1} \rightarrow \infty}{\stackrel{\mathcal{D}}{\longrightarrow}} P
$$


Now, as in the proof of Theorem 5, (9), (13) and (14) together with [3, Theorem 4.2] give the relation

$$
\mathbf{X}_{T}(s) \underset{T \rightarrow \infty}{\stackrel{\mathcal{D}}{\longrightarrow}} P
$$

which shows that the measure $P_{T}$ converges weakly to $P$ as $T \rightarrow \infty$. Moreover, (15) shows that the measure $P$ is independent of the subsequence $\left\{P_{n_{1}}\right\}$. This, the relative compactness of $\left\{P_{n}\right\}$ and [3, Theorem 2.3] give the relation

$$
\mathbf{X}_{n}(s) \underset{n \rightarrow \infty}{\stackrel{\mathcal{D}}{\longrightarrow}} P
$$

Now define

$$
\widehat{\mathbf{X}}_{T}(s)=\left(\zeta\left(s+i T \theta, \alpha_{1}, \omega_{1} ; \mathfrak{a}_{1}\right), \ldots, \zeta\left(s+i T \theta, \alpha_{r}, \omega_{r} ; \mathfrak{a}_{r}\right)\right) .
$$

Then the same arguments as applied for $\widehat{\mathbf{X}}_{T, n}(s)$ and $\widehat{\mathbf{X}}_{T}(s)$ as well as relations (12) and (16) allow us to obtain that the measure $\widehat{P}_{T}$, for almost all $\omega \in \Omega^{r}$, also converges weakly to the measure $P$ as $T \rightarrow \infty$.

Now it remains to give the explicit form of the measure $P$. For this, we apply some statements from ergodic theory. Define $a_{\tau, j}=\left\{\left(m+\alpha_{j}\right)^{-i \tau} \mid m \in \mathbb{N}_{0}\right\}, \tau \in \mathbb{R}$, $j=1, \ldots, r$. Then, for each $j=1, \ldots, r,\left\{a_{\tau, j} \mid \tau \in \mathbb{R}\right\}$ is a one-parameter group. Let $\left\{\Phi_{\tau} \mid \tau \in \mathbb{R}\right\}=\left\{\left(\varphi_{\tau, 1}, \ldots, \varphi_{\tau, r}\right) \mid \tau \in \mathbb{R}\right\}$, where $\varphi_{\tau, j}\left(\omega_{j}\right)=a_{\tau, j} \omega_{j}, \omega_{j} \in \Omega_{j}$, $j=1, \ldots, r$. Then $\left\{\Phi_{\tau} \mid \tau \in \mathbb{R}\right\}$ is a one-parameter group of measurable measurepreserving transformations on $\Omega^{r}$.

We shall prove that the one-parameter group $\left\{\Phi_{\tau} \mid \tau \in \mathbb{R}\right\}$ is ergodic. A set $A \in \mathcal{B}\left(\Omega^{r}\right)$ is called an invariant set with respect to the group $\left\{\Phi_{\tau} \mid \tau \in \mathbb{R}\right\}$ if, for each $\tau$, the sets $A$ and $A_{\tau}=\Phi_{\tau}(A)$ differ by a set of zero $m_{H r}$-measure. In other words, $m_{H r}\left(A \triangle A_{\tau}\right)=0$, where $A \triangle A_{\tau}$ denotes the symmetric difference. We have to prove that the $\sigma$-field of invariant sets of $\left\{\Phi_{\tau} \mid \tau \in \mathbb{R}\right\}$ consists only of sets having $m_{H r}$-measure equal to 0 or 1 .

Let $\chi: \Omega^{r} \rightarrow \gamma$ be a character of $\Omega^{r}$. In the proof of Lemma 3 we have seen that

$$
\chi(\boldsymbol{\omega})=\prod_{j=1}^{r} \prod_{m=0}^{\infty} \omega_{j}^{k_{m j}}(m), \quad \boldsymbol{\omega}=\left(\omega_{1}, \ldots, \omega_{j}\right) \in \Omega^{r},
$$

where only a finite number of integers $k_{m j}$ are distinct from zero. Suppose that $\chi$ is a nontrivial character of $\Omega^{r}$. Then we have that

$$
\begin{aligned}
\chi\left(a_{\tau, 1}, \ldots, a_{\tau, r}\right) & =\prod_{j=1}^{r} \prod_{m=0}^{\infty}\left(m+\alpha_{j}\right)^{-i \tau k_{m j}} \\
& =\exp \left\{-i \tau \sum_{j=1}^{r} \sum_{m=0}^{\infty} k_{m j} \log \left(m+\alpha_{j}\right)\right\},
\end{aligned}
$$


where only a finite number of $k_{m j}$ are distinct from zero. By the hypothesis of the theorem, the numbers $\alpha_{1}, \ldots, \alpha_{r}$ are algebraically independent, therefore

$$
\sum_{j=1}^{r} \sum_{m=0}^{\infty} k_{m j} \log \left(m+\alpha_{j}\right) \neq 0 .
$$

Hence, there exists $\tau_{0} \neq 0$ such that

$$
\chi\left(a_{\tau_{0}, 1}, \ldots, a_{\tau_{0}, r}\right) \neq 1
$$

Let $A \in \mathcal{B}\left(\Omega^{r}\right)$ be an invariant set with respect to $\left\{\Phi_{\tau} \mid \tau \in \mathbb{R}\right\}$. Denote by $I_{A}$ the indicator (characteristic) function of $A$. Then, for each $\tau \in \mathbb{R}$ and almost all $\omega \in \Omega^{r}$,

$$
I_{A}\left(\mathfrak{a}_{\tau} \boldsymbol{\omega}\right)=I_{A}(\boldsymbol{\omega})
$$

where $\mathfrak{a}_{\tau}=\left(a_{\tau, 1}, \ldots, a_{\tau, r}\right)$. Denote by $\widehat{g}$ the Fourier transform of the function $g$. Then in view of (18)

$$
\begin{aligned}
\widehat{I}_{A}(\chi) & =\int_{\Omega^{r}} \chi(\boldsymbol{\omega}) I_{A}(\boldsymbol{\omega}) m_{H r}(d \boldsymbol{\omega}) \\
& =\int_{\Omega^{r}} \chi(\boldsymbol{\omega}) I_{A}\left(\mathfrak{a}_{\tau_{0}} \boldsymbol{\omega}\right) m_{H r}(d \boldsymbol{\omega}) \\
& =\chi\left(\mathfrak{a}_{\tau_{0}}\right) \int_{\Omega^{r}} \chi(\boldsymbol{\omega}) I_{A}(\boldsymbol{\omega}) m_{H r}(d \boldsymbol{\omega}) \\
& =\chi\left(\mathfrak{a}_{\tau_{0}}\right) \widehat{I}_{A}(\chi) .
\end{aligned}
$$

Hence, from (17) we have that $\widehat{I}_{A}(\chi)=0$ for all nontrivial characters of $\Omega^{r}$.

Now let $\chi_{0}$ be the trivial character of $\Omega\left(\chi_{0}(\omega) \equiv 1, \omega \in \Omega^{r}\right)$. Suppose that $\widehat{I}_{A}\left(\chi_{0}\right)=u$. Then, using

$$
\int_{\Omega^{r}} \chi(\boldsymbol{\omega}) m_{H r}(d \boldsymbol{\omega}) \begin{cases}1 & \text { if } \chi=\chi_{0} \\ 0 & \text { if } \chi \neq \chi_{0}\end{cases}
$$

and the equalities $\widehat{I}_{A}(\chi)=0, \widehat{I}_{A}\left(\chi_{0}\right)=u$, we find that, for each character $\chi$ of $\Omega^{r}$,

$$
\widehat{I}_{A}(\chi)=u \int_{\Omega^{r}} \chi(\omega) m_{H r}(d \omega)=u \widehat{1}(\chi)=\widehat{u}(\chi) .
$$

The function $I_{A}(\omega)$ is uniquely determined by its Fourier transform $\widehat{I}_{A}(\chi)$. Therefore, from (19) we have that $I_{A}(\omega)=u$ for almost all $\omega \in \Omega^{r}$. However, $I_{A}(\omega)$ is the indicator function. Hence, $u=0$ or 1 , that is, $I_{A}(\omega)=0$ for almost all $\omega \in \Omega^{r}$ or $I_{A}(\omega)=1$ for almost all $\omega \in \Omega^{r}$. Therefore, $m_{H r}(A)=0$ or 1 , and we have that the one-parameter group $\left\{\Phi_{\tau} \mid \tau \in \mathbb{R}\right\}$ is ergodic. 
Now we are ready to complete the proof of Theorem 2. The argument is standard. Let $A \in \mathcal{B}\left(H^{r}(D)\right)$ be a continuity set of the measure $P$. Then the weak convergence of the measure $\widehat{P}_{T}$ and [3, Theorem 2.1] show that

$$
\lim _{T \rightarrow \infty} v_{T}\left(\left(\zeta\left(s+i \tau, \alpha_{1}, \omega_{1} ; \mathfrak{a}_{1}\right), \ldots, \zeta\left(s+i \tau, \alpha_{r}, \omega_{r} ; \mathfrak{a}_{r}\right)\right) \in A\right)=P(A),
$$

for almost all $\omega \in \Omega^{r}$. Next, we fix the set $A$ and define a random variable $X$ on the probability space $\left(\Omega^{r}, \mathcal{B}\left(\Omega^{r}\right), m_{H r}\right)$ by

$$
X(\boldsymbol{\omega})= \begin{cases}1 & \text { if } \zeta(s, \boldsymbol{\alpha}, \boldsymbol{\omega} ; \mathfrak{a}) \in A \\ 0 & \text { if } \zeta(s, \boldsymbol{\alpha}, \boldsymbol{\omega} ; \mathfrak{a}) \notin A .\end{cases}
$$

Let $\mathbb{E}(X)$ denote the expectation of $X$. Then

$$
\mathbb{E}(X)=\int_{\Omega^{r}} X d m_{H r}=m_{H r}\left(\boldsymbol{\omega} \in \Omega^{r}: \zeta(s, \boldsymbol{\alpha}, \boldsymbol{\omega} ; \mathfrak{a}) \in A\right)=P_{\zeta}(A) .
$$

Since the one-parameter group $\left\{\Phi_{\tau} \mid \tau \in \mathbb{R}\right\}$ is ergodic, the random process $X\left(\Phi_{\tau}(\boldsymbol{\omega})\right)$ is also ergodic. Therefore, the classical Birkhoff-Khinchine theorem yields

$$
\lim _{T \rightarrow \infty} \frac{1}{T} \int_{0}^{T} X\left(\Phi_{\tau}(\boldsymbol{\omega})\right) d \tau=\mathbb{E}(X),
$$

for almost all $\omega \in \Omega^{r}$. However, the definitions of $X$ and $\left\{\Phi_{\tau} \mid \tau \in \mathbb{R}\right\}$ show that

$$
\frac{1}{T} \int_{0}^{T} X\left(\Phi_{\tau}(\boldsymbol{\omega})\right) d \tau=v_{T}(\zeta(s+i \tau, \boldsymbol{\alpha}, \boldsymbol{\omega} ; \mathfrak{a}) \in A) .
$$

From this and (21), (22) we deduce that

$$
\lim _{T \rightarrow \infty} \nu_{T}(\zeta(s+i \tau, \boldsymbol{\alpha}, \boldsymbol{\omega} ; \mathfrak{a}) \in A)=P_{\zeta}(A),
$$

for almost all $\omega \in \Omega^{r}$. Therefore, in view of (20), $P(A)=P_{\zeta}(A)$ for continuity sets $A$ of the measure $P$. Hence, $P(A)=P_{\zeta}(A)$ for all $A \in \mathcal{B}\left(H^{r}(D)\right)$, and the theorem is proved.

\section{Proof of Theorem 1}

We begin with the support of the measure $P_{\zeta}$. We recall that the support of $P_{\zeta}$ is a minimal closed set $S_{P_{\zeta}} \subseteq H^{r}(D)$ such that $P_{\zeta}\left(S_{P_{\zeta}}\right)=1$. Denote by $S_{X}$ the support of the random element $X$.

For convenience, we state two lemmas from [19].

LEMMA 6. Let $\left\{\mathbf{X}_{m}\right\}$ be a sequence of independent $H^{r}(D)$-valued random elements such that the series

$$
\sum_{m=1}^{\infty} \mathbf{X}_{m}
$$


converges almost surely. Then the support of the sum of the latter series is the closure of the set of all $f \in H^{r}(D)$ which may be written as a convergent series

$$
\boldsymbol{f}=\sum_{m=1}^{\infty} \boldsymbol{f}_{m}, \quad \boldsymbol{f}_{m} \in S_{X_{m}} .
$$

This lemma is a partial case of [19, Lemma 5].

LEMMA 7. Let $\left\{\boldsymbol{f}_{m}\right\}=\left\{f_{1 m}, \ldots, f_{r m}\right\}$ be a sequence in $H^{r}(D)$ which satisfies:

(a) if $\mu_{1}, \ldots, \mu_{n}$ are complex measures on $(\mathbb{C}, \mathcal{B}(\mathbb{C}))$ with compact supports contained in $D$ such that

$$
\sum_{m=1}^{\infty}\left|\sum_{j=1}^{r} \int_{\mathbb{C}} f_{j m} d \mu_{j}\right|<\infty,
$$

then

$$
\int_{\mathbb{C}} s^{l} d \mu_{j}(s)=0,
$$

for $j=1, \ldots, r, l=0,1,2, \ldots$;

(b) the series

$$
\sum_{m=1}^{\infty} f_{m}
$$

converges in $D$;

(c) for any compact $K_{j} \subset D, j=1, \ldots, r$,

$$
\sum_{m=1}^{\infty} \sum_{j=1}^{r} \sup _{s \in K_{j}}\left|f_{j m}(s)\right|^{2}<\infty .
$$

Then the set of all convergent series

$$
\sum_{m=1}^{\infty} a_{m} \boldsymbol{f}_{m}
$$

with $a_{m} \in \gamma$ is dense in $H^{r}(D)$.

This lemma is a partial case of [19, Lemma 6].

LEMMA 8. Suppose that the numbers $\alpha_{1}, \ldots, \alpha_{r}$ are algebraically independent over $\mathbb{Q}$ and that $\operatorname{rank}(A)=r$. Then the support of the measure $P_{\zeta}$ is the whole of $H^{r}(D)$.

ProOF. For $\omega \in \Omega$, define

$$
\widehat{\zeta}(s, \boldsymbol{\alpha}, \omega ; \mathfrak{a})=\left(\zeta\left(s, \alpha_{1}, \omega ; \mathfrak{a}_{1}\right), \ldots, \zeta\left(s, \alpha_{r}, \omega ; \mathfrak{a}_{r}\right)\right), \quad s \in D,
$$


where

$$
\zeta\left(s, \alpha_{j}, \omega ; \mathfrak{a}_{j}\right)=\sum_{m=0}^{\infty} \frac{a_{m j} \omega(m)}{\left(m+\alpha_{j}\right)^{s}}, \quad j=1, \ldots, r .
$$

Let $P_{\widehat{\zeta}}$ denote the distribution of the random element $\widehat{\zeta}(s, \boldsymbol{\alpha}, \omega ; \mathfrak{a})$. Obviously, $S_{P_{\zeta}} \supseteq S_{P_{\widehat{\zeta}}}$. Hence, it suffices to prove that $S_{P_{\widehat{\zeta}}}=H^{r}(D)$.

The support of each random variable $\omega(m)$ is $\gamma$. Hence, the support of the $H(D)$ valued random element

$$
\frac{a_{m j} \omega(m)}{\left(m+\alpha_{j}\right)^{s}}
$$

is the set

$$
\left\{g \in H(D) \mid g(s)=\frac{a_{m j} a}{\left(m+\alpha_{j}\right)^{s}} \text { with } a \in \gamma\right\}, \quad m \in \mathbb{N}_{0}, j=1, \ldots, r .
$$

By the definition $\left\{\omega(m) \mid m \in \mathbb{N}_{0}\right\}$ is a sequence of independent random variables defined on $\left(\Omega, \mathcal{B}(\Omega), m_{H}\right)$. Therefore,

$$
\left\{\frac{a_{m j} \omega(m)}{\left(m+\alpha_{j}\right)^{s}} \mid m \in \mathbb{N}_{0}\right\}
$$

is a sequence of independent $H(D)$-valued random elements defined on $\left(\Omega, \mathcal{B}(\Omega), m_{H}\right)$, and for the support $S_{P_{\widehat{\zeta}}}$ Lemma 6 can be applied. Thus, we have that $S_{P_{\widehat{\zeta}}}$ is the closure of the set of all convergent series

$$
\sum_{m=0}^{\infty}\left(\frac{a_{m 1} a_{m}}{\left(m+\alpha_{1}\right)^{s}}, \ldots, \frac{a_{m r} a_{m}}{\left(m+\alpha_{r}\right)^{s}}\right),
$$

with $a_{m} \in \gamma, m \in \mathbb{N}_{0}$. To study the set of series (23), we shall apply Lemma 7 .

Since $\widehat{\boldsymbol{\zeta}}(s, \boldsymbol{\alpha}, \omega ; \mathfrak{a})$ is an $H^{r}(D)$-valued random element, there exists a sequence $\left\{b_{m} \mid b_{m} \in \gamma, m \in \mathbb{N}_{0}\right\}$ such that the series

$$
\sum_{m=0}^{\infty}\left(\frac{a_{m 1} b_{m}}{\left(m+\alpha_{1}\right)^{s}}, \ldots, \frac{a_{m r} b_{m}}{\left(m+\alpha_{r}\right)^{s}}\right)
$$

converges in $H^{r}(D)$. Moreover, since $\left|a_{m j}\right| \leq C$ with a certain $C>0, m \in \mathbb{N}_{0}$, $j=1, \ldots, r$, for every compact subset $K$ of $D$,

$$
\sum_{m=0}^{\infty} \sum_{j=1}^{r} \sup _{s \in K} \frac{\left|a_{m j}\right|^{2}}{\left(m+\alpha_{j}\right)^{2 \sigma}}<\infty .
$$

Now let $\mu_{1}, \ldots, \mu_{r}$ be complex measures on $(\mathbb{C}, \mathcal{B}(\mathbb{C}))$ with compact supports contained in $D$ and such that

$$
\sum_{m=0}^{\infty}\left|\sum_{j=1}^{r} \int_{\mathbb{C}} \frac{a_{m j} b_{m}}{\left(m+\alpha_{j}\right)^{s}} d \mu_{j}(s)\right|<\infty .
$$


Using the estimate

$$
\left(m+\alpha_{j}\right)^{-s}=m^{-s}+m^{-1-\sigma} O\left(|s| e^{O(|s|)}\right),
$$

we can rewrite (24) in the form

$$
\sum_{m=0}^{\infty}\left|\sum_{j=1}^{r} \int_{\mathbb{C}} \frac{a_{m j}}{m^{s}} d \mu_{j}(s)\right|<\infty .
$$

In view of the periodicity of $a_{m j}$, this implies, for $l=1, \ldots, k$,

$$
\sum_{\substack{m=0 \\ m \equiv l(\bmod k)}}^{\infty}\left|\sum_{j=1}^{r} \frac{a_{l j}}{m^{s}} d \mu_{j}(s)\right|<\infty .
$$

Now we set

$$
\nu_{l}(A)=\sum_{j=1}^{r} a_{l j} \mu_{j}(A), \quad A \in \mathcal{B}(\mathbb{C}), l=1, \ldots, k .
$$

Then $v_{1}, \ldots, v_{k}$ are again complex measures on $(\mathbb{C}, \mathcal{B}(\mathbb{C}))$ with compact supports contained in $D$, and by (25)

$$
\sum_{\substack{m=0 \\ m \equiv l(\bmod k)}}^{\infty}\left|\int_{\mathbb{C}} m^{-s} d v_{l}(s)\right|<\infty, \quad l=1, \ldots, k .
$$

If we define

$$
\varrho_{l}(z)=\int_{\mathbb{C}} e^{-s z} d \nu_{l}(s), \quad z \in \mathbb{C}, l=1, \ldots, k,
$$

this gives

$$
\sum_{\substack{m=0 \\ m \equiv l(\bmod k)}}^{\infty}\left|\varrho_{l}(\log m)\right|<\infty, \quad l=1, \ldots, k
$$

For $l=1, \ldots, k, \varrho_{l}(z)$ is an entire function of exponential type, therefore, by [10, Lemma 6.4.10], either $\varrho_{l}(z) \equiv 0$, or

$$
\limsup _{x \rightarrow \infty} \frac{\log \left|\varrho_{l}(x)\right|}{x}>-1, \quad l=1, \ldots, k .
$$

If we assume that the latter inequality is true for some $l$, then by [17, Lemma 5], for this $l$,

$$
\sum_{\substack{m=0 \\ m \equiv l(\bmod k)}}^{\infty}\left|\varrho_{l}(\log m)\right|=\infty
$$


and this contradicts (26). Thus, we obtain that $\varrho_{l}(z) \equiv 0, l=1, \ldots, k$, or, by the definition of $v_{l}$,

$$
\sum_{j=1}^{r} a_{l j} \int_{\mathbb{C}} e^{-s z} d \mu_{j}(s) \equiv 0, \quad l=1, \ldots, k .
$$

Now we apply the rank condition. Since $\operatorname{rank}(A)=r$, we find that

$$
\int_{\mathbb{C}} e^{-s z} d \mu_{j}(s) \equiv 0, \quad j=1, \ldots, r,
$$

and, by differentiation, after putting $z=0$, we obtain that

$$
\int_{\mathbb{C}} s^{l} d \mu_{j}(s) \equiv 0
$$

for all $l \in \mathbb{N}_{0}, j=1, \ldots, r$. Thus, we obtained that all hypotheses of Lemma 7 are satisfied for the set of all convergent series

$$
\sum_{m=0}^{\infty}\left(\frac{a_{m 1} b_{m} a_{m}}{\left(m+\alpha_{1}\right)^{s}}, \ldots, \frac{a_{m r} b_{m} a_{m}}{\left(m+\alpha_{r}\right)^{s}}\right),
$$

with $a_{m} \in \gamma$. Hence, the latter set is dense in $H^{r}(D)$. Therefore, the set of all convergent series (23) is also dense in $H^{r}(D)$, and this completes the proof of lemma.

Proof of Theorem 1. First we suppose that the functions $f_{1}(s), \ldots, f_{r}(s)$ are analytically continuable to the strip $D$. Let

$$
G=\left\{\left(g_{1}, \ldots, g_{r}\right) \in H^{r}(D)\left|\sup _{1 \leq j \leq r} \sup _{s \in K_{j}}\right| g_{j}(s)-f_{j}(s) \mid<\varepsilon\right\} .
$$

Clearly, the set $G$ is open. Moreover, $S_{P_{\zeta}}$ consists of all $\mathbf{g} \in H^{r}(D)$ such that for every neighbourhood $\mathcal{G}$ of $\mathbf{g}$ the inequality $P_{\zeta}(\mathcal{G})>0$ is satisfied. Therefore, by Lemma 8 , $P_{\zeta}(G)>0$. Hence, from [3, Theorems 2 and 2.1] we obtain

$$
\liminf _{T \rightarrow \infty} v_{T}\left(\sup _{1 \leq j \leq r} \sup _{s \in K_{j}}\left|\zeta\left(s+i \tau, \alpha_{j} ; \mathfrak{a}_{j}\right)-f_{j}(s)\right|<\varepsilon\right) \geq P_{\zeta}(G)>0 .
$$

Now let the functions $f_{1}(s), \ldots, f_{r}(s)$ satisfy the hypotheses of Theorem 1 . By the Mergelyan theorem (see, for example, [26]) there exists polynomials $p_{1}(s), \ldots, p_{r}(s)$ such that

$$
\sup _{1 \leq j \leq r} \sup _{s \in K_{j}}\left|f_{j}(s)-p_{j}(s)\right|<\varepsilon / 2 .
$$

Since the polynomials $p_{1}(s), \ldots, p_{r}(s)$ are entire functions, by the first part of the proof we have that

$$
\liminf _{T \rightarrow \infty} \nu_{T}\left(\sup _{1 \leq j \leq r} \sup _{s \in K_{j}}\left|\zeta\left(s+i \tau, \alpha_{j} ; \mathfrak{a}_{j}\right)-p_{j}(s)\right|<\varepsilon / 2\right)>0 .
$$


However, by (27)

$$
\begin{aligned}
& \left\{\sup _{1 \leq j \leq r} \sup _{s \in K_{j}}\left|\zeta\left(s+i \tau, \alpha_{j} ; \mathfrak{a}_{j}\right)-f_{j}(s)\right|<\varepsilon\right\} \\
& \quad \supseteq\left\{\sup _{1 \leq j \leq r} \sup _{s \in K_{j}}\left|\zeta\left(s+i \tau, \alpha_{j} ; \mathfrak{a}_{j}\right)-p_{j}(s)\right|<\varepsilon\right\} .
\end{aligned}
$$

This together with (28) give the assertion of the theorem.

\section{Acknowledgement}

The authors are grateful to an anonymous referee for useful remarks and suggestions.

\section{References}

[1] B. Bagchi, 'The statistical behaviour and universality properties of the Riemann zeta-function and other allied Dirichlet series', PhD Thesis, Indian Statistical Institute, Calcutta, 1981.

[2] _ 'Joint universality theorem for Dirichlet L-functions', Math. Z. 181 (1982), 319-334.

[3] P. Billingsley, Convergence of Probability Measures (J. Wiley \& Sons, New York, 1968).

[4] J. Genys and A. Laurinčikas, 'Value distribution of general Dirichlet series', Liet. Mat. Rink. 44(2) (2004), 181-195 (in Russian); Lithuanian Math. J. 44(2) (2004), 145-156.

[5] S. M. Gonek, 'Analytic properties of zeta and $L$-functions', PhD Thesis, University of Michigan, 1979.

[6] K.-G. Grosse-Erdmann, 'Universal families and hypercyclic operators', Bull. Amer. Math. Soc. 36 (1999), 345-381.

[7] H. Heyer, Probability Measures on Locally Compact Groups (Springer, Berlin, 1977).

[8] A. Javtokas and A. Laurinčikas, 'On the periodic Hurwitz zeta-function', Hardy-Ramanujan J. 29 (2006), 18-36.

[9] _ 'The universality of the periodic Hurwitz zeta-function', Integral Transforms Spec. Funct. 17(10) (2006), 711-722.

[10] A. Laurinčikas, Limit Theorems for the Riemann Zeta-function (Kluwer Academic, Dordrecht, 1996).

[11] _ ' 'On the zeros of linear combinations of Matsumoto zeta-functions', Liet. Mat. Rink. 38 (1998), 185-204 (in Russian). Lithuanian. Math. J. 38 (1998), 144-159.

[12] _ 'The joint universality for general Dirichlet series', Ann. Univ. Sci. Budapest. Sect. Comput. 22 (2003), 235-251.

[13] _ _ 'The universality of zeta-functions', Acta Appl. Math. 78 (2003), 251-271.

[14] _ 'The joint universality of Dirichlet series', Proc. Sci. Seminar Faculty of Physics and Math., Šiauliai Univ. 7 (2004), 33-44.

[15] — - 'The joint universality for periodic Hurwitz zeta-functions', Analysis 26 (2006), 419-428.

[16] _ ' 'The Voronin-type theorem for periodic Hurwitz zeta-functions', Sb. Math. 198(1-2) (2007), 231-242.

[17] A. Laurinčikas and R. Garunkštis, The Lerch Zeta-function (Kluwer Academic, Dordrecht, 2002).

[18] A. Laurinčikas and K. Matsumoto, 'The joint universality and the functional independence for Lerch zeta-functions', Nagoya Math. J. 157 (2000), 211-227.

[19] _ 'The joint universality of zeta-functions attached to certain cusp forms', Proc. Sci. Seminar Faculty of Physics and Math., Siauliai Univ. 5 (2002), 58-75.

[20] _ _ 'The joint universality of twisted automorphic L-functions', J. Math. Soc. Japan 56(3) (2004), 923-939. 
[21] K. Matsumoto, 'Probabilistic value-distribution theory of zeta functions', Sugaku Expositions 17(1) (2004), 51-71.

[22] J. Steuding, Value-distribution of L-functions, Lecture Notes in Mathematics, 1877 (Springer, Berlin, 2007).

[23] R. Šleževičienė, 'The joint universality for twists of Dirichlet series with multiplicative coefficients', in: Analytic and Probability Methods in Number Theory, Proc. 3rd Int. Conf. in Honor of J. Kubilius, Palanga, 2001 (eds. A. Dubickas et al.) (TEV, Vilnius, 2002), pp. 303-319.

[24] S. M. Voronin, 'Theorem on the universality of the Riemann zeta-function', Math. USSR Izv. 9 (1975), 443-453.

[25] _ 'On the functional independence of Dirichlet L-functions', Acta Arith. 27 (1975), 493-503 (in Russian).

[26] J. L. Walsh, 'Interpolation and approximation by rational functions in the complex domain', Amer. Math. Soc. Colloq. Publ. 20 (1960).

A. JAVTOKAS, Department of Math. and Informatics, Vilnius University, Naugarduko 24, 03225 Vilnius, Lithuania

e-mail: ajavtokas@math.com

A. LAURINČIKAS, Department of Math. and Informatics, Vilnius University, Naugarduko 24, 03225 Vilnius, Lithuania

e-mail: antanas.laurincikas@maf.vu.lt 